\author{
Military Technical College \\ Kobry El-Kobbah, \\ Cairo, Egypt.
}

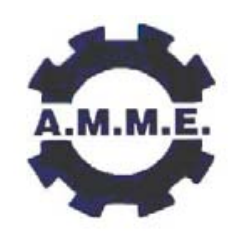

$13^{\text {th }}$ International Conference on Applied Mechanics and Mechanical Engineering.

\title{
EVALUATION OF CHARACTERISTICS OF TRACKED VEHICLES TORSION BARS
}

\author{
SALEM $^{*}$ A.M. and SALAHUDDIEN ${ }^{* *}$ T.
}

\begin{abstract}
Suspension system of high mobility tracked vehicles is often fitted with torsion bars and hydraulic shock absorbers. Characteristics of these elements have a great effect on shocks and vibration resulting from drive of vehicles over irregular terrain. This study is concerned with evaluation of the stiffness of torsion bar of Armored Personnel Carrier M113 and how it can be affected by road configuration and vehicle speed. A complete test rig equipped with a complete measuring system has been designed and manufactured to measure the moment acting on torsion bar and the corresponding twist angle. The measured parameters are used to evaluate vehicle suspension characteristics. The frequency and amplitude of an arbitrary rigid terrain profile with constant vehicle speed are introduced in the test rig by adapting the rotating speed and the profile of rig driving cam. Design and simulation of the test rig were carried out by the aid of ADAMS program.
\end{abstract}

\section{KEY WORDS}

Tracked vehicle, suspension, torsion bar, spring

\footnotetext{
** Egyptian Armed Forces
} 


\section{INTRODUCTION}

The earliest tracked vehicles had fixed suspension system with no movement. This unsatisfactory situation was improved with leaf spring suspensions adopted from agricultural machinery, but even these had very limited travel. In the 1930s, the use of coil springs inside vehicle's hull has been allowed by redirecting the direction of travel using a bell crank. High-mobility tracked vehicles, such as battle tanks and armored personnel carriers, are designed for drive over rough terrain surfaces. The mobility performance of these vehicles is often limited by the operator's endurance to withstand the transmitted shocks and vibrations, and his ability to maintain control.

High-mobility tracked vehicles are generally fitted with passive suspension systems utilizing torsion bars and shock absorbers to attenuate the terrain-induced shocks and vibrations. The torsion bar replaces the coil spring in old tracked vehicles as it performs spring actions with improved characteristics [1-3]. In order to evaluate suspension characteristics for such vehicles, it is necessary to evaluate characteristics of suspension elements. Parameters of these elements comprise torsion bars stiffness and shock absorber damping coefficient. The torsion bar connects the road wheel with vehicle hull through a rocker arm that rotates about the axis of torsion bar while the shock absorber connects front and rear road wheels with hull body [4]. The maximum allowable speed of vehicle is primarily influenced by the roughness of terrain, and suspension system design. When driving a vehicle over uneven road, the elastic elements, represented by torsion bars, are twisted resulting in hull vibration. Characteristics of such vibration depend on torsion bar stiffness and shock absorber damping coefficient.

A torsion bar is a rod that resists twisting and has a strong tendency to return to its original position when twisted. In tracked vehicles the torsion bar is a long spring-steel element with one end held rigidly to the vehicle hull and the other end is twisted by a lever connected to the road wheel axle. It is used for optimization of driving comfort and driving safety. Therefore, it is necessary to identify characteristics of torsion bar under real conditions. The objective of this work is to evaluate the characteristics of suspension system through measuring of torsion bar stiffness and compare it with the theoretical value. The dynamic behavior of torsion bar is studied by using the constructed test rig which provides a sinusoidal excitation to the bar with the same working conditions of vehicle and road profile.

\section{DETERMINATION OF TORSION BAR CHARACTERISTICS}

Torsion bar characteristics are the variation of acting moment on bar with the corresponding twist angle. They are determined theoretically using mathematical relations derived from the basic form of twisted bar and then they are evaluated experimentally using the designed and manufactured test rig. Suspension characteristics of tracked vehicles is the relationship between the vertical force $P_{w}$ acting on the road wheel and the corresponding vertical displacement $f_{w}$ resulting from such force as shown in figure 1. 


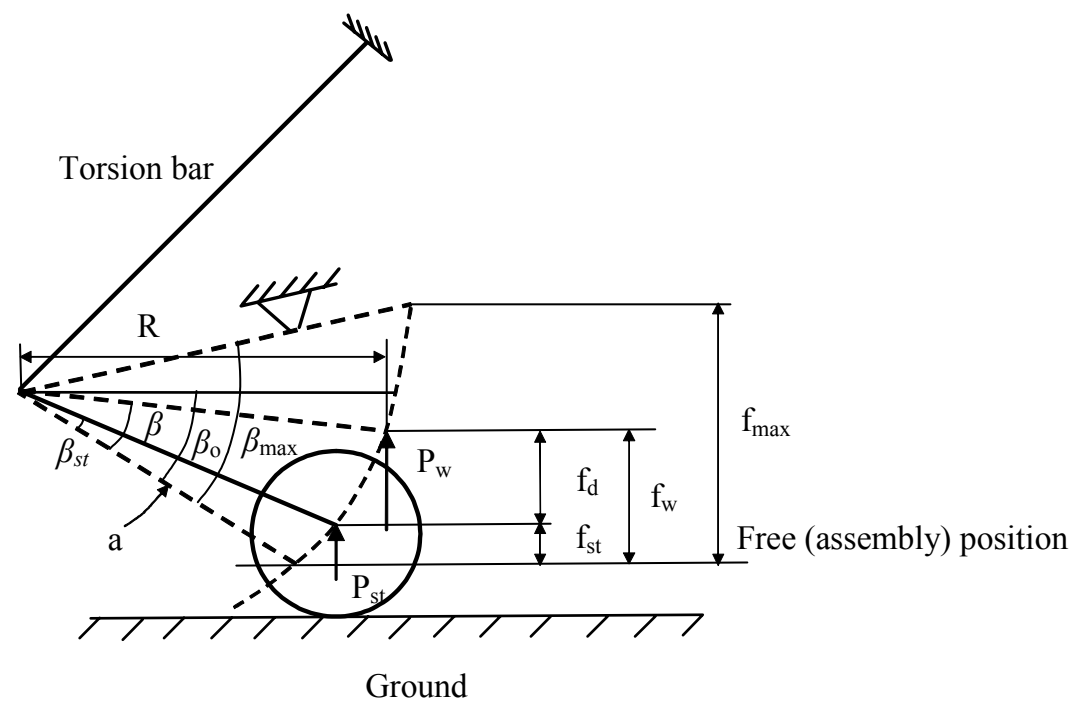

Figure 1: Schematic of road wheel and torsion bar connection

The torsion bar is acted upon by a torsional moment $M_{t}$ resulting from the load exerted on the road wheel and transmitted through the axle arm [5]. This moment is correlated to the resulted twist angle $\beta$ by the following relationship:

$$
M_{t}=\frac{G I}{L} \beta=C \beta
$$
$\beta$ : $\quad$ angle of twist of torsion bar
$L: \quad$ active length of torsion bar
l: $\quad$ polar moment of inertia of torsion bar section, $\quad I=\frac{\pi d^{4}}{32}$
d: $\quad$ diameter of torsion bar
C: $\quad$ torsion bar stiffness
$G$ : $\quad$ shear modulus of elasticity of torsion bar material

It follows from the previous equation that the torsional stiffness $C=\frac{M_{t}}{\beta}$ is constant and it represents the characteristic parameter of torsion bar. For the M113: $d=3.66 \mathrm{~cm}, L=$ $1.51 \mathrm{~m}, a=33.5 \mathrm{~cm}$. The M113 torsion bar characteristic is shown in figure 2.

From figure 1 the moment, $M_{t}$, is determined as:

$$
M_{t}=P_{w} R \quad, \quad R=\operatorname{acos}\left(\beta_{o}-\beta\right)
$$

Therefore, from both relations of the moment, $P_{w}$ is found as:

$$
P_{w}=\frac{G I}{L} \frac{\beta}{\operatorname{acos}\left(\beta_{o}-\beta\right)}
$$

Also, the vertical displacement of road wheel $f_{w}$ is given as: 


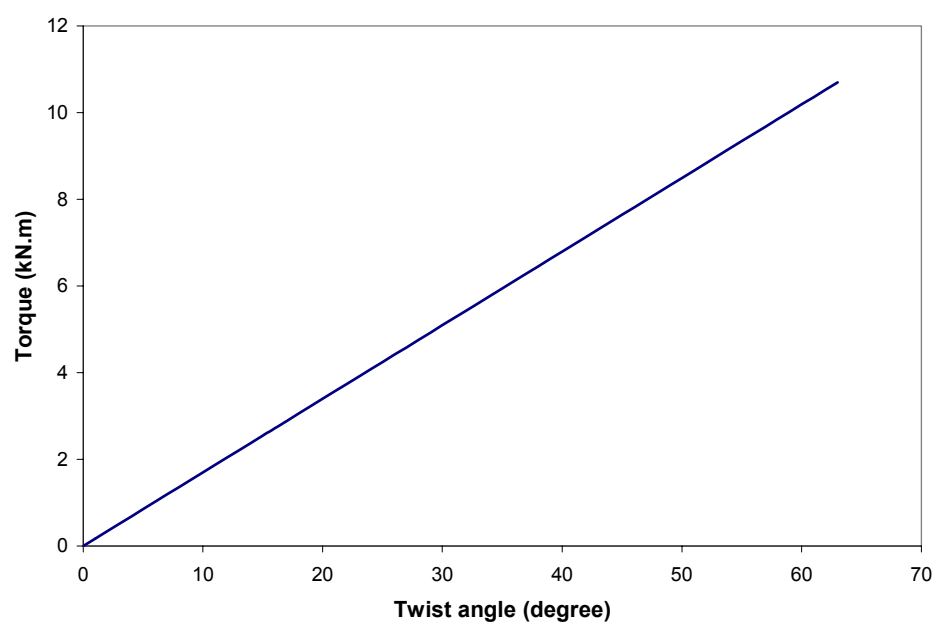

Figure 2: M113 torsion bar characteristics.

$$
f_{w}=a \sin \beta_{o}-a \sin \left(\beta_{o}-\beta\right)
$$

where $\beta_{0}$ is the angle of inclination of the rocker arm when the road wheel is fully relieved and $a$ is the length of rocker arm. For various values of angle $\beta$, it is possible to calculate different values of $P_{w}$ and $f_{w}$ and to express graphically the relationship:

$$
P_{w}=f\left(f_{w}\right)
$$

Figure 3 demonstrates the suspension characteristics of M113 where they are found to be generally nonlinear. The suspension spring stiffness (or reduced stiffness of torsion bar) $C_{w}$ is determined as the first derivative of function $P_{w}=f\left(f_{w}\right)$ with respect to $f_{w}$ :

$$
C_{w}=\frac{d P_{w}}{d f_{w}}
$$

It is possible to investigate the stiffness $C_{w}$ graphically by dividing the change of the acting force on road wheel $P_{w}$ by the change of the corresponding displacement $f_{w}$ so that:

$$
C_{w}=\frac{\Delta P_{w}}{\Delta f_{w}}
$$

However, the stiffness of suspension spring can be taken in suspension calculations as constant and equal to its average value. From the figure, it is seen that the average value of such stiffness is about $133(\mathrm{kN} / \mathrm{m})$.

\section{TEST RIG AND MEASURING SYSTEM}

A mechanical test rig was designed and constructed for measuring the stiffness of APC M113 torsion bar [6-7]. The test rig enables putting the bar in the same working conditions and with the same profile of road. This means that the torsion bar is excited with a sinusoidal wave of the same amplitude and frequency of the road taking into consideration the vehicle speed. The frequency of excitation, $\omega$ of torsion bar can be calculated from the following relation: 


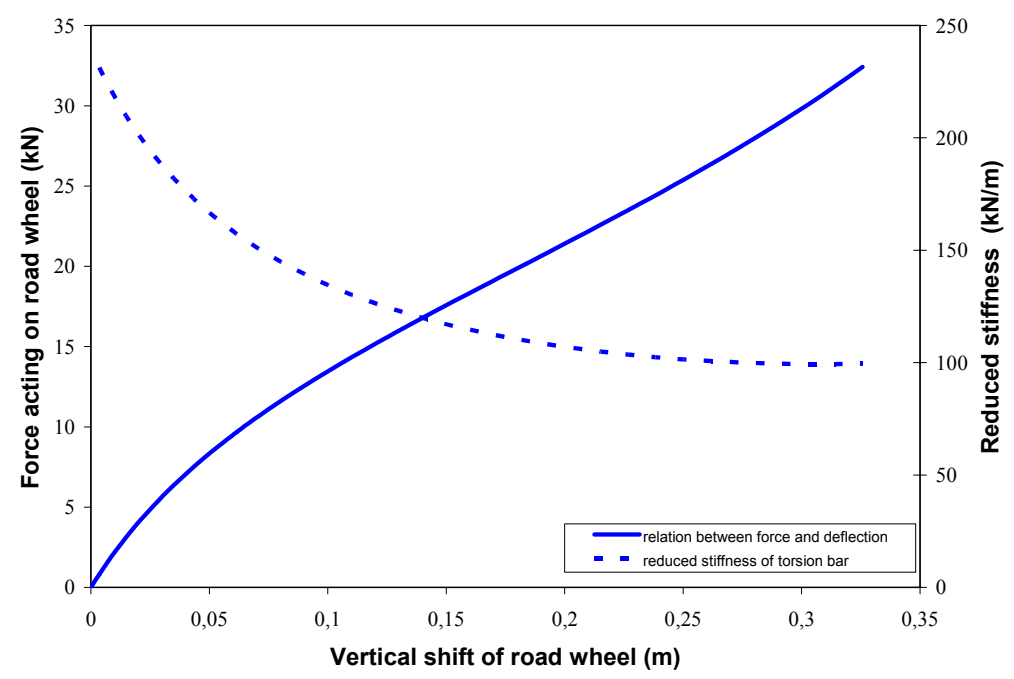

Figure 3: Suspension characteristics of APC M113.

$$
\omega=\frac{2 \pi v}{a} t
$$

where $v$ is the vehicle speed and $a$ is the road wave length. A typical configuration of road way is shown in figure 4 . The excitation is achieved by twisting and rebound of torsion bar with a cam mechanism. The test rig is equipped with measuring instruments for getting the applied torque and the corresponding twist angle.

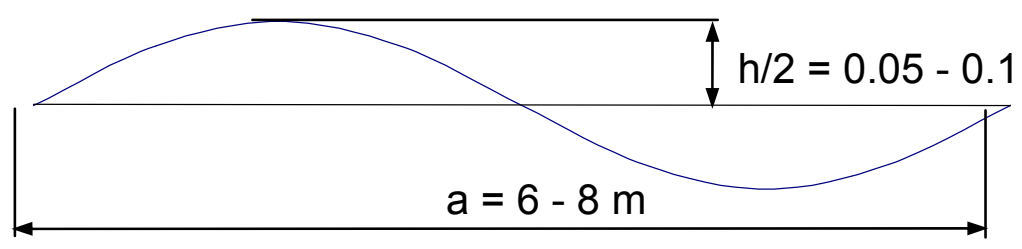

Figure 4: Typical road configuration for tracked vehicles

The test rig comprises a frame structure, driving system and measuring system as shown in figure 5.

The rig structure consists of a steel frame formed by $I$ and $C$ beams. The free end of the torsion bar is twisted by a cam mechanism while the other end is held stationary on the frame structure. A 20HP AC motor coupled with a reduction gear box of $80 \mathrm{rpm}$ output is fixed on a steel base plate welded to the frame.

The motor drives a rotating shaft on two bearings fixed to base plate, through a couple of spur gears set to change the speed of the shaft. A special cam provides the same road profile is fixed on the middle of the rotating shaft and drives the torsion bar through the rocker arm by means of roller. Figure 6 shows a complete description of the drive mechanism of torsion bar. 


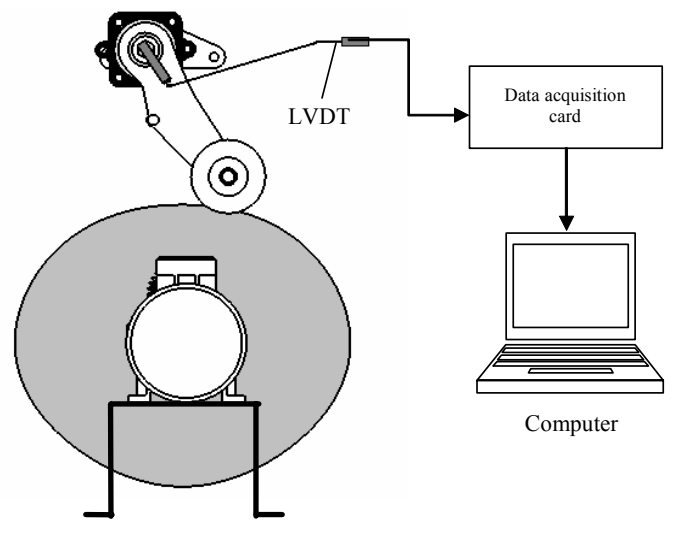

a. connection of LVDT

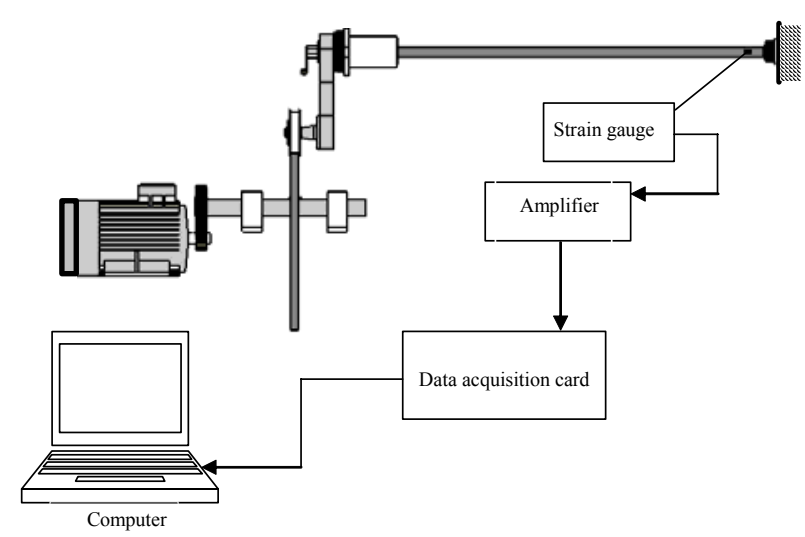

b. connection of strain gauges

Figure 5: Schematic drawing of test rig with measuring system
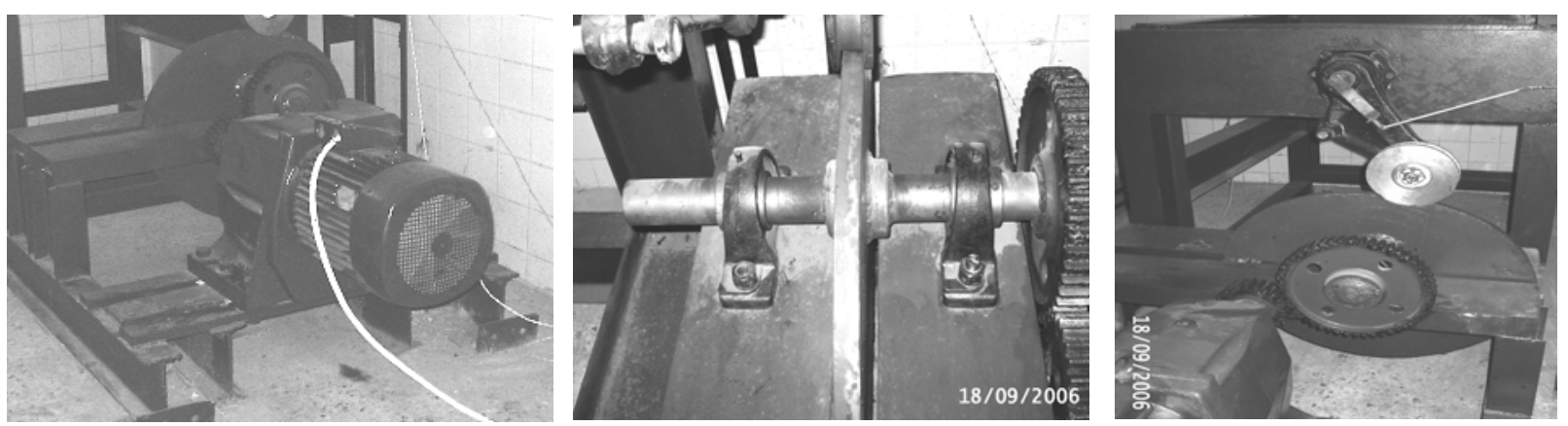

Figure 6: Drive mechanism of the torsion bar

Design and simulation of the cam mechanism is carried out using Automotive Dynamic Analysis of Mechanical System (ADAMS) software as shown in figure 7. The program is used to determine the angle of rotation of the axle and hence the twisting angle of torsion bar.
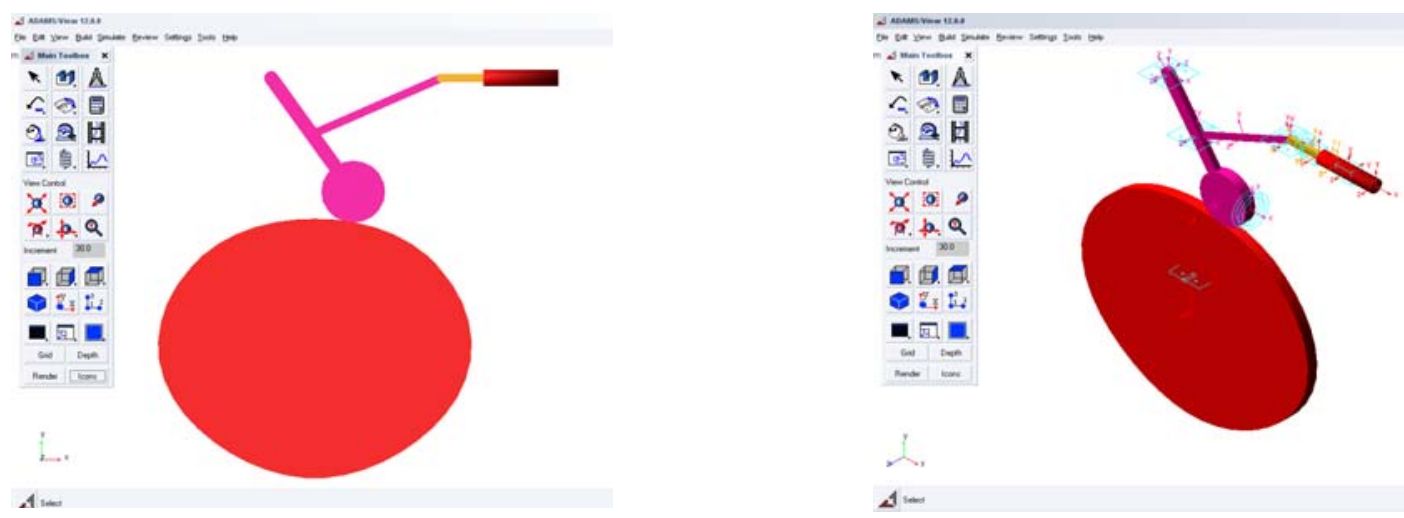

Figure 7: Simulation of cam mechanism using ADAMS software 


\section{EXPERIMENTAL RESULTS}

The experimental results are obtained after measuring the applied torque on torsion bar and the corresponding twist angle. The twisting angle is measured by means of linear variable differential transducer (LVDT) while the applied torque is measured by a system of strain gauges.

\section{Measuring System}

The linear variable differential transducer (LVDT) type WA100 with displacement (010) $\mathrm{cm}$ is used to measure the twisting angle of torsion bar. It gives the displacement of a piston connected to the axle arm by means of a slider crank mechanism so that the distance $S$ represents the stroke of LVDT. Figure 8 shows the connection of LVDT with the slider crank mechanism. The displacement of the piston is converted to a twisting angle by mathematical relations as follows:
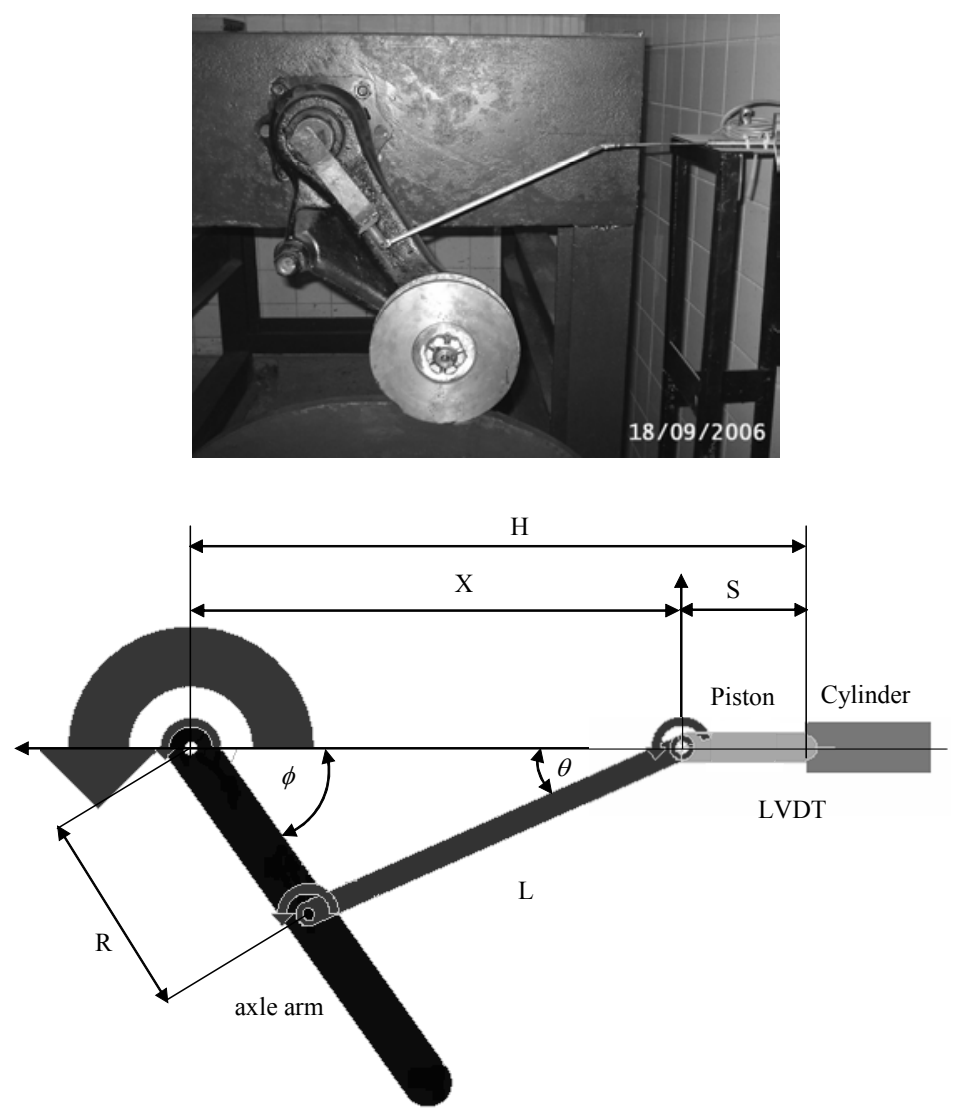

Figure 8: LVDT connection with slider crank mechanism

The victor equation for the closure of the slider crank mechanism is:

where:

$$
R+L-X=0 \quad, \quad X=H-S, \quad S=0
$$

$\mathrm{R}$ : $\quad$ Length of the axle arm in connection with the slider crank mechanism

$\mathrm{L}$ : $\quad$ Length of connecting rod

S: $\quad$ Stroke of the piston 
This is equivalent to the scalar equations:

$$
\begin{gathered}
R \cos \phi+L \cos \theta-X=0 \\
R \sin \phi-L \sin \theta=0
\end{gathered}
$$

Since the parameter $S$ is adjusted to be $S=10 \mathrm{~cm}$ (maximum deflection of LVDT), then determination of unknowns $\theta, \phi$ can be done by solving the above equations:

$$
\begin{gathered}
L \sin \theta=R \sin \phi \\
X=R \cos \phi+L \cos \theta=R \cos \phi+L \sqrt{1-(R / L)^{2} \sin ^{2} \phi}
\end{gathered}
$$

Taking the values of $L=332 \mathrm{~mm}, \mathrm{R}=166 \mathrm{~mm}$ and substituting:

$$
\sin ^{2} \phi=1-\cos ^{2} \phi
$$

The angle of rotation of the torsion bar can be obtained as:

$$
\phi=\cos ^{-1}\left(\frac{X}{33.2}-\frac{24.9}{X}\right)
$$

The signal from LVDT is transferred to a PC through a DAQ (data acquisition card D-1 $\mathrm{NI}$ USB-6211) and then the corresponding displacement is obtained using a LabVIEW program. The variation of the measured voltage with time can be obtained and, hence the variation of LVDT displacement with time can be also obtained. Thus, the change of twist angle of the torsion bar with time can be calculated from the above relation for each value of LVDT displacement.

A full bridge strain gauge (No. kfg-10-120-c1-11) attached near to the fixed end of torsion bar is used to measure the developed torque through measuring of the deformation in torsion bar. The signal developed from the strain gauge is sent to a strain gauge amplifier (No.435-692) where it would be enlarged, and then the signal is transferred to a PC where it is transformed to torsional strain.

Figure 9 shows the connection of strain gauges used in strain measurements. The output of strain-gauge bridge is expressed as an output voltage (mV/V or $\mu \mathrm{V} / \mathrm{V})$ against the bridge voltage. The relation between the strain quantity and the output voltage has the following form:

$$
\gamma_{0}=\frac{4 e_{0}}{E K_{S}}
$$

where:

$\gamma_{0}: \quad$ torsional strain

$E: \quad$ input voltage (5.46 volt)

$e_{0}: \quad$ output voltage

$k_{s}$ : gauge factor (2.11) 


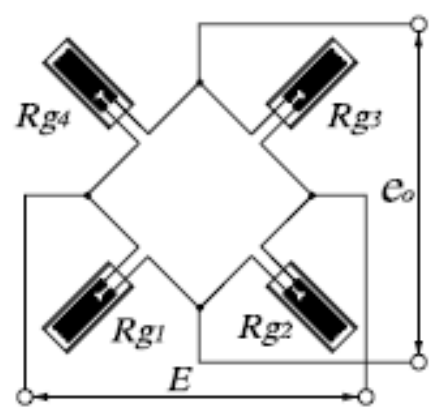

Figure 9: Strain Gauge Bridge

After measuring the strain, the torsional stress can be found by the stress strain relation:

$$
\tau=\gamma_{0} G_{s h}
$$

and the twisting torque can be calculated by the following relation:

$$
T=\frac{\pi d^{3}}{16} \tau
$$

\section{Measuring of Shear Modulus of Elasticity}

The value of shear modulus of elasticity of the torsion bar is usually taken as an approximated value $G_{s h}=83.4 \mathrm{GPa}$. For proper calculations, it was necessary to measure accurately its value. For this purpose a standard specimen was prepared from an original M113A2 torsion bar with dimensions shown in figure 10 according to the ASTM standard.

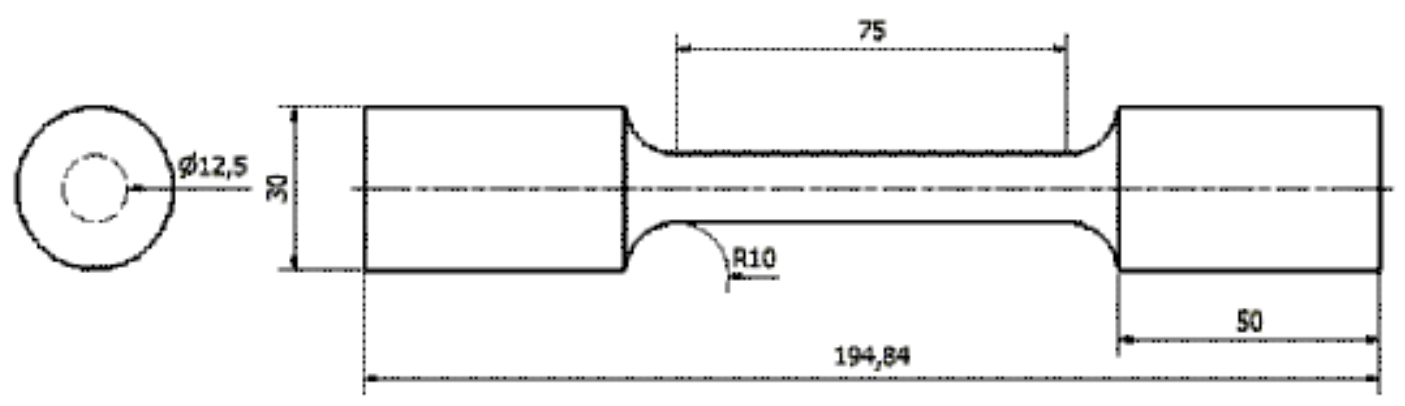

Figure 10: ASTM Specimen

The purpose of the test is to measure Young's modulus of elasticity of the torsion bar, and then calculate the shear modulus of elasticity. The test is performed on a special MTS (Material Testing System) shown in figure 11. The stress-strain diagram obtained from the standard tensile test of the specimen in the elastic range is shown in figure 12. 


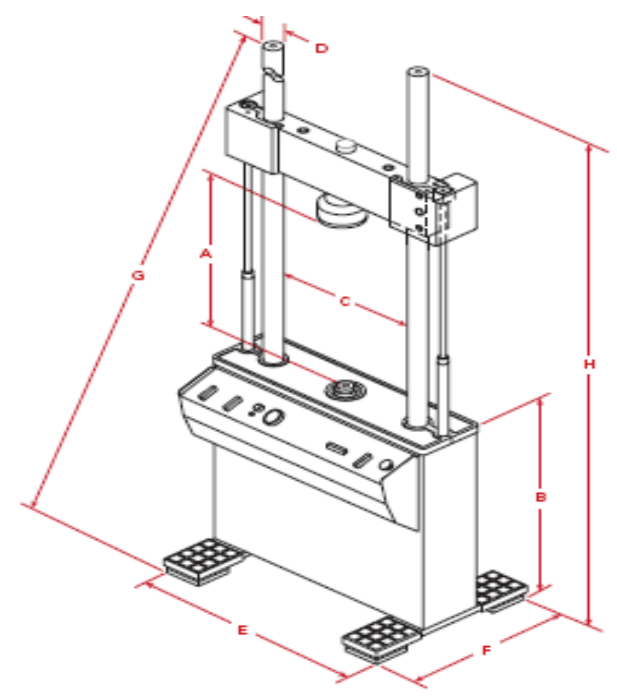

Figure 11: Load unit assembly

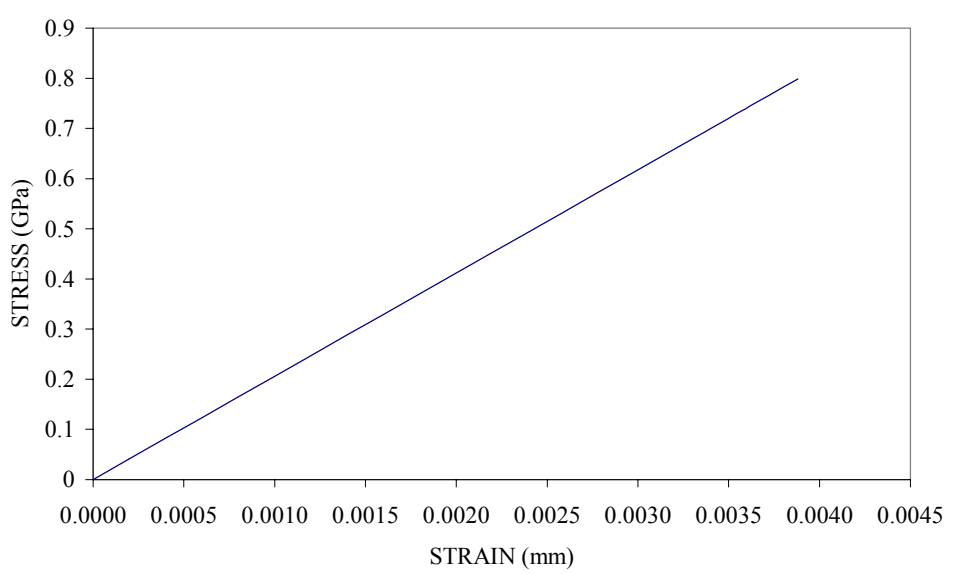

Figure 12: Stress-Strain diagram (elastic range) of M113 torsion bar

The value of Young's modulus $E$ obtained from the test and the value of Poisson's ratio $v$ of torsion bar material are used to calculate the shear modulus of elasticity of the torsion bar so that:

$$
G_{s h}=\frac{E}{2(1+v)}
$$

From the previous diagram it can be found that the Young's Modulus of Elasticity $E=205.88 \mathrm{GPa}$. By knowing Poisson's ratio for steel $(v=0.29)$, the value of the shear modulus of elasticity will be:

$$
G_{s h}=79.8 \mathrm{GPa}
$$

The suspension characteristics of M113 considering the measured shear modulus of elasticity of torsion bar is calculated and represented in figure 13. The figure compares between the suspension characteristics calculated with the assumed value of shear modulus of elasticity and that with the measured one. It is shown from the figure that the difference between the two cases is very small which mean that it is permitted with good agreement to take in calculation of torsion bar stiffness the assumed value of 


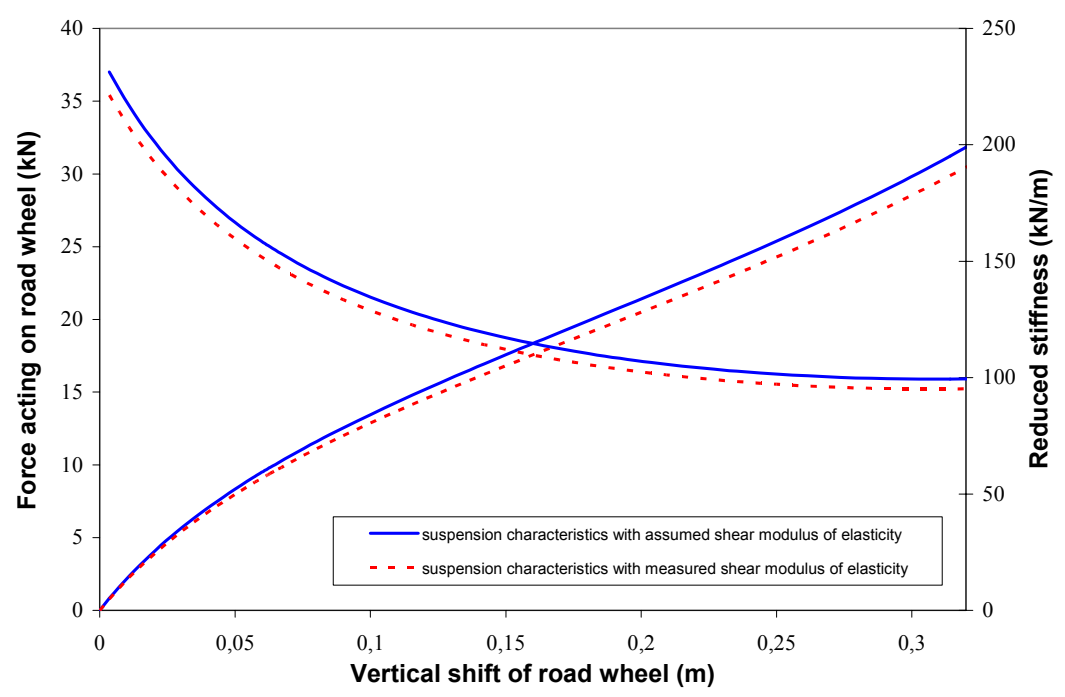

Figure 13: Suspension characteristics of APC M113

shear modulus of elasticity. From the figure, it can be found that the average value of suspension stiffness (reduced stiffness of torsion bar) of M113 is about $127 \mathrm{kN} / \mathrm{m}$.

\section{Test Results}

Variation of the twist angle of torsion bar measured using LVDT with time is shown in figure 14. It is shown from the figure that the maximum twisted angle of torsion bar, realized by the designed cam, is about $45^{\circ}$. When the twist angle of torsion bar reaches its maximum value, the rotating cam returns the torsion bar to its initial position and the cycle is repeated for few seconds. Figure 15 shows the variation of the measured torque using full strain bridge with time. At each interval of time the torque and the corresponding angle are recorded and the relation between them is illustrated in figure 16 for twisting and rebound stroke of torsion bar. The figure shows that the relation of the applied torque on the torsion bar and the resulting twist angle has a linear trend with small amount of nonlinearity.

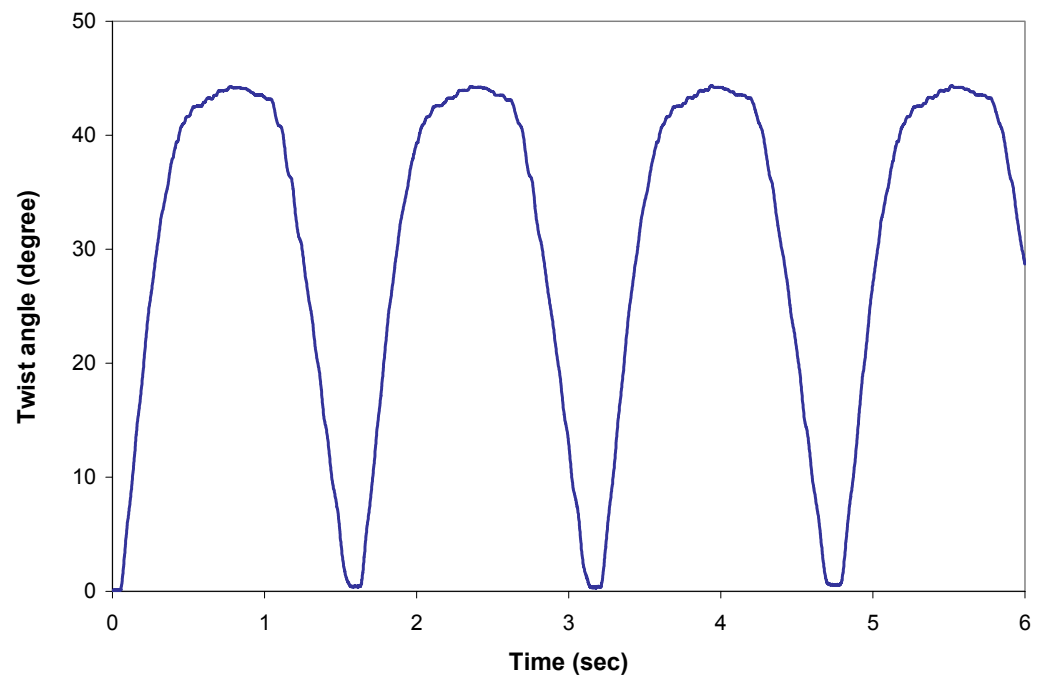

Figure 14: Variation of measured twist angle with time 


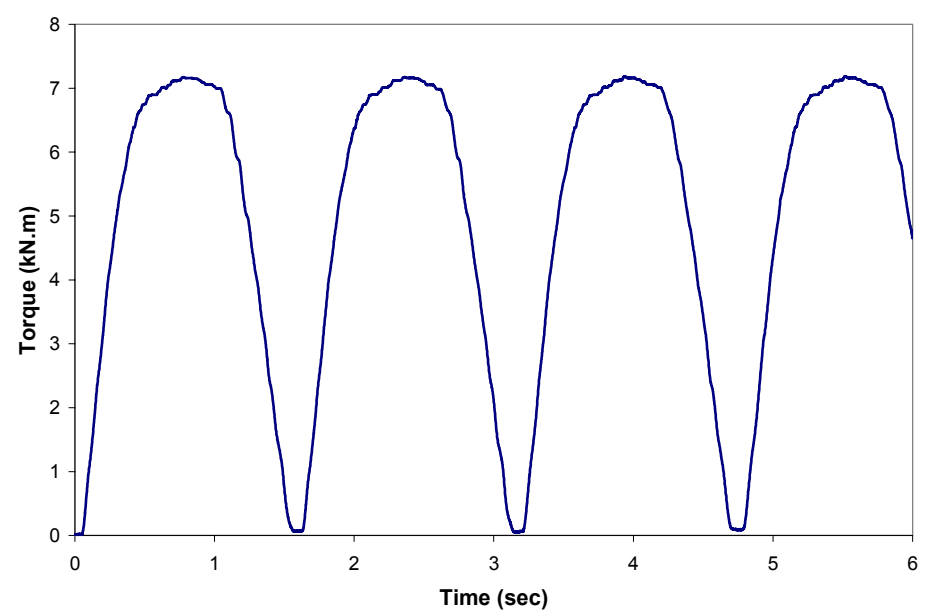

Figure 15: Variation of measured torque with time

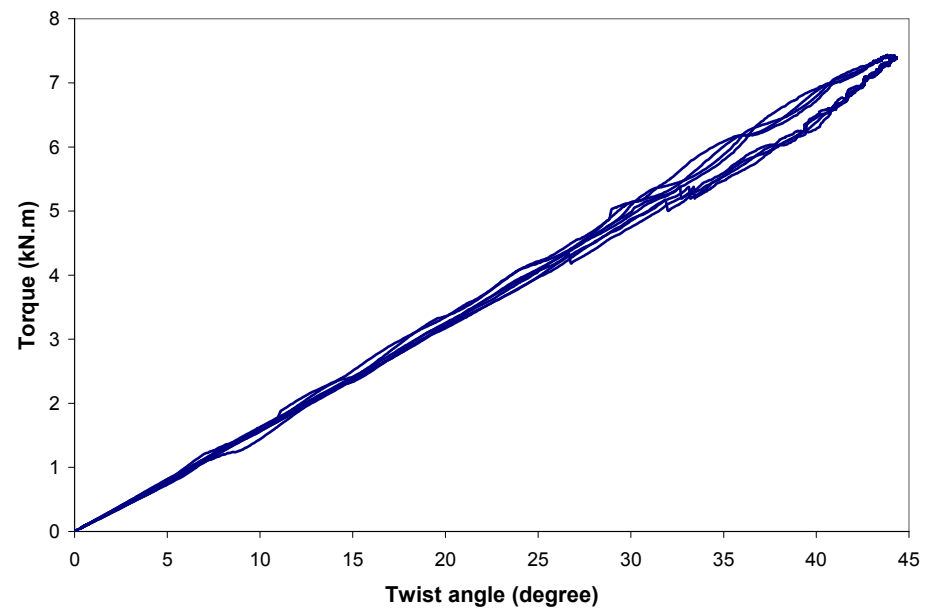

Figure 16: Measured characteristics of torsion bar

Figure 17 compare the twisting angle measured on the test rig using LVDT system with that simulated by ADAMS. It is shown that the measured results are correlated with the simulated one.

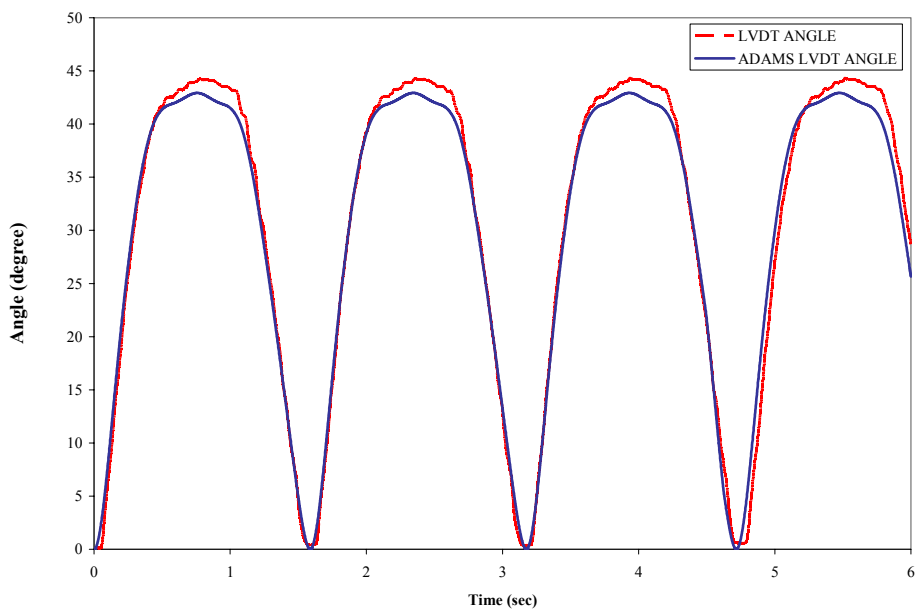

Figure 17: Comparison between twisting angle using ADAMS and LVDT. 
Figure 18 illustrates the suspension characteristics of the torsion bar measured from the test rig while figure 19 compares the calculated characteristics with the measured one. It is shown that the measured characteristic is coming close to the calculated one. This means that during evaluation of the vehicle response due to its drive over irregular roads, it can be used with good agreement the value taken from calculations.

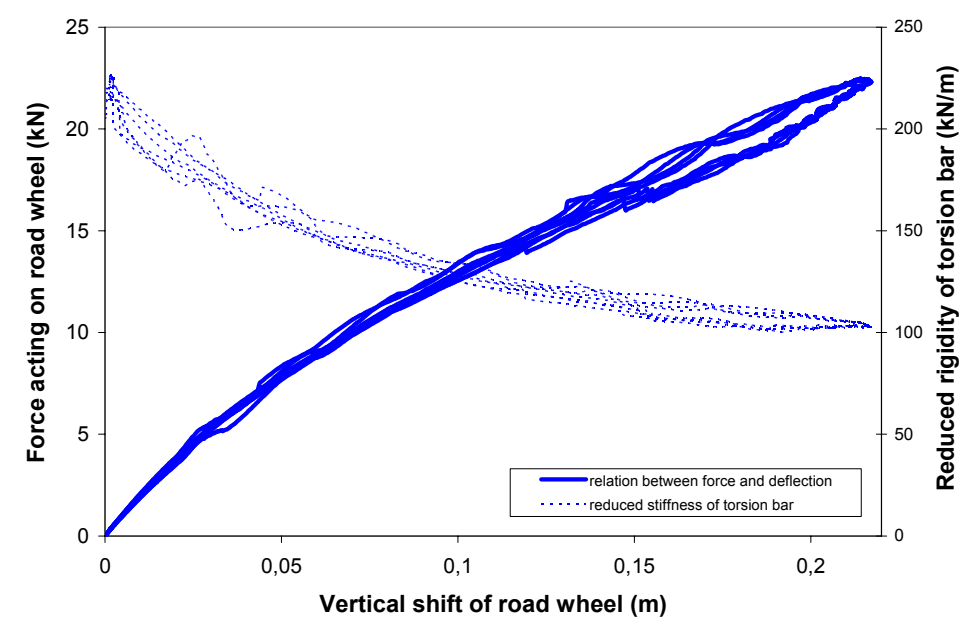

Figure 18: Measured characteristics of suspension

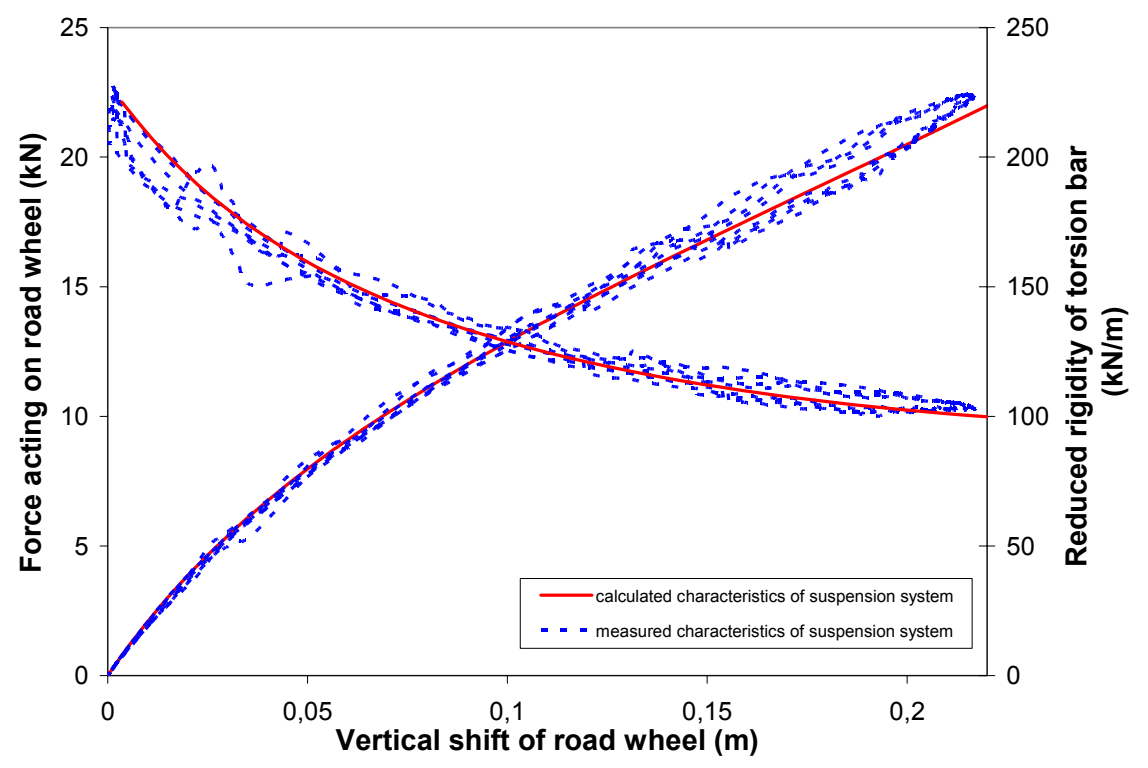

Figure 19: Comparison between measured and calculated suspension characteristics

\section{CONCLUSIONS}

1. Evaluation of torsion bar stiffness of APC M113 has been realized experimentally using a new designed and manufactured test rig with proper accuracy. The new test rig can be used for measuring torsion bar stiffness of different types of tracked vehicle torsion bars with different road configurations and different vehicle speeds. It was found from the experimental measurements that the calculated torsion bar 
characteristics have a little difference from the measured one due the assumptions and the approximated values used in calculations.

2. A tensile test had been carried out to measure the real value of shear modulus of elasticity of APC M113 torsion bar using Material Testing System (MTS). It was found from calculation that the nominal value of suspension stiffness of APC M113 is about $127(\mathrm{kN} / \mathrm{m})$. This value is calculated using the measured value of shear modulus of elasticity.

3. The torsional stiffness of a uniform rod is in general, dependent on the cross-section diameter, the length, and the shear modulus of elasticity of the rod. Thus, the torsion bar spring rate can be varied by appropriately changing any of the previous parameters.

\section{REFERENCES}

[1] A spatial motion analysis model of tracked vehicles with torsion bar type suspension, J.Yamakawa, K. Watanabe Department of Mechanical Engineering, The National Defense Academy, 1-10-20 Hashirimizu, Yokosuka 239-8686, Japan, 2004

[2] Assessment of Tracked Vehicle Suspension System Using A Validated Computer Simulation Model, ANIL DHIR and SESHADRI SANKAR, Journal of Terramechanics, Vol. 32, No. 3, pp. 127-149, 1995

[3] Investigation into the effect of suspension characteristics and design parameters on the performance of tracked vehicles using an advanced computer simulation model, J Y Wong, PhD, DSc, CEng, FIMechE and J Preston-Thomas, BEng. Proceedings 1988

[4] Printed lectures of tanks department, construction of tracked vehicles, part I, chair of tanks, M.T.C, Cairo, Egypt, 1995.

[5] Printed lecture of tanks department, Theory of suspension, chair of tanks, M.T.C., Cairo, Egypt

[6] MACHINE DESIGN ACAD Approach ANDREW D. DIMAROGONAS Copright 2001 by John Wiley \& Sons, Inc.

[7] MECHANICAL ENGINEERING DESIGN, Joseph Edward Shigley, Copyright @ 1986 